\title{
Study of Liquid Crystal on Silicon Displays for Their Application in Digital Holography
}

\author{
Angel Lizana ${ }^{1}$, Laura Lobato ${ }^{1}$, Andrés Márquez², Claudio Iemmi³ \\ Ignacio Moreno ${ }^{4}$, Juan Campos ${ }^{1}$ and María J. Yzuel ${ }^{1}$ \\ 1 Universitat Autònoma de Barcelona \\ ${ }^{2}$ Universidad de Alicante \\ ${ }^{3}$ Universidad de Buenos Aires \\ ${ }^{4}$ Universidad Miguel Hernández \\ 1,2,4Spain \\ ${ }^{3}$ Argentina
}

\section{Introduction}

Due to the significant capability of Liquid Crystal Displays (LCDs) to spatially manipulate the phase information of an incident light beam, this technology is been widely applied in a large number of optical applications. Nowadays, they are employed as Spatial Light Modulators (SLMs) in many areas, as for instance, in Optical Image Processing (Liu et al., 1985), in Holography Data Storage (Coufal et al., 2000), in Programmable Adaptive Optics (Dou \& Giles, 1995), in Medical Optics (Twietmeyer et al., 2008), or in Diffractive Optics (Márquez et al., 2005), among others.

Recently, a new type of reflective LCDs, the Liquid Crystals on Silicon (LCoS) displays, have awakened a great interest due to their specific technical characteristics, which in general, are superior in many aspects to the ones provided by transmissive LCDs (Lee et al., 2004; Wu \& Yang, 2005). For instance, as LCoS displays work in reflection, the light impinging such devices performs a double pass through the LC cell, leading to a larger phase modulation than that related to transmissive LCDs with the same thickness. This greater phase modulation capability allows LCoS displays to become very suitable devices for digital holography applications, as for instance, for laser beam shaping (Dickey et al., 2005; Rodrigo et al., 2011) or for optical micro-particle manipulation (Ashkin, 2006).

To maximize the efficiency of digital holograms generated by using LCoS displays, it is required to apply a suitable methodology for optimizing the performance of these devices when generating the specific phase and amplitude distributions. Nowadays, there exist different theoretical models proposed to improve the performance of LCDs (Azzam \& Bashara, 1972; Gagnon, 1981; Márquez et. al., 2001). In general, most of these models are based on mathematical formalisms describing fully polarized light, as the Jones formalism (Jones, 1941) or the Berreman formalism (Berreman, 1972). However, some authors have discovered that LCoS displays may introduce non-negligible values of effective depolarization at the reflected beam (Lizana et al., 2008a; Márquez et al., 2008; Wolfe \& Chipman, 2006), which are originated by the electrical addressing schemes applied in these devices (Hermerschmidt et al., 2007). This effective depolarization depends on the incident 
angle (Lizana et al., 2009; Verma et al., 2010), the wavelength (Lizana et al., 2008c) and the state of the polarization of the incident beam (Márquez et al., 2008). In this situation, the application of an optimizing method that includes the unpolarized light contribution observed when working with LCoS display becomes mandatory.

In Ref. (Lizana et al., 2008b), another undesired phenomenon originated by the electrical addressing schemes applied in LCoS displays is reported. We refer to the time-fluctuations of the phase phenomenon, which may notably degrade the efficiency of the digital holograms generated with LCoS displays (Lizana et al., 2008b). Thus, to maximize the efficiency of digital holograms addressed to these devices, different ways to reduce the undesired influence of this damaging phenomenon must be applied.

This Chapter presents a study based on LCoS displays which can be useful as a guideline to optimize the performance of these devices for the generation of digital holograms. In section 2, a characterization and optimization methodology, based on Mueller-Stokes (M-S) formalism is described. This methodology considers the effective depolarization values observed in LCoS displays because the M-S formalism is able to describe fully polarized light, partial polarized light and unpolarized light contributions. In section 3, experimental evidences of the time-fluctuations of the phase phenomenon are presented and its effects on the generation of digital holograms are reviewed. In section 4, a method based on the minimum Euclidean distance principle, devised to reduce the undesired influence of this phenomenon is proposed and experimentally tested by analyzing the efficiency of different optimized digital holograms addressed to an LCoS display. Finally, conclusions are given in section 5 .

\section{Liquid Crystal on Silicon displays characterization and optimization methodology}

In this section, we propose a characterization-optimization method, based on a combination of the Mueller-Stokes (M-S) formalism (Parke, 1949) and the Jones formalism (Jones, 1941), which is able to predict and optimize the intensity and the phase response of LCoS displays. The characterization of the LCoS display is conducted as a function of the addressed gray level (which is related to the applied voltages), by experimentally calibrating its corresponding Mueller matrices, which are able to describe depolarizing samples. In this situation, the intensity response of the LCoS display can be predicted but since Mueller matrices are calculated from intensity measurements, no information about the phase modulation response is obtained. As Jones matrices contain the required phase information, a conversion from Mueller matrices to Jones matrices is applied. To perform a proper conversion, a polar decomposition (Lu \& Chipman, 1996; Foldyna et al., 2009) of the experimentally obtained Mueller matrices is carried on.

Once the intensity and phase response of the LCoS display can be predicted, an optimization procedure is applied, in order to get the desired LCoS display behaviour, i.e. to work as amplitude-only modulator or as phase-only modulator. In next subsections, the characterization and optimization methodology is described in detail and some results are provided.

\subsection{LCoS display Mueller matrix characterization: Intensity prediction}

The LCoS display under analysis is a commercial Philips LCoS model X97c3A0, sold as the kit LC-R2500 by HoloEye Systems. This prototype presents a monochrome reflective 
Twisted Nematic (TN) LCoS display of $2.46 \mathrm{~cm}$ diagonal, in which the twist angle of LC is 45 degrees. The resolution of the display is XGA (1024x768 pixels), with a fill factor of $93 \%$. The pixels are square and they are separated by a distance (center to center) of $19 \mu \mathrm{m}$. In addition, the device provides digitally controlled gray scales with 256 gray levels (8 bits).

To determine the Mueller matrix of the TNLCoS display, the set-up sketched in Fig. 1 is used.

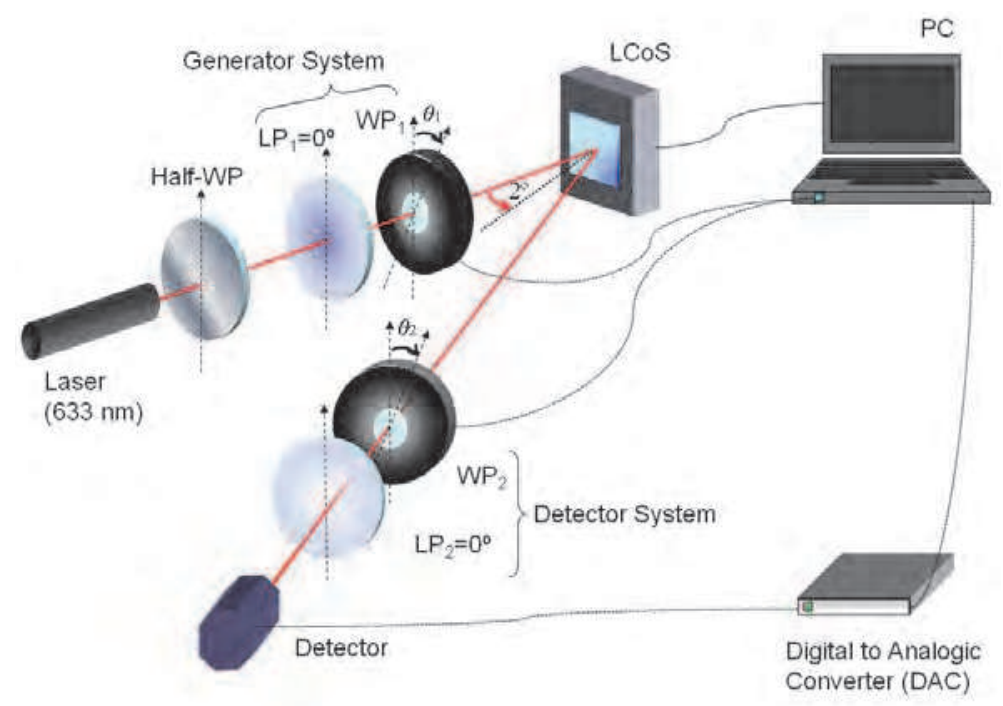

Fig. 1. Mueller matrix characterization set-up.

The TNLCoS display is illuminated by a linear polarized He-Ne laser source with a wavelength equal to $633 \mathrm{~nm}$. In the incident beam, just following the laser source, is set a Half-Waveplate (HWP) that allows us to control the intensity of light transmitted by the linear polarizer $\mathrm{LP}_{1}$. After the HWP element, a Polarization State Generator (PSG), which is formed by a linear polarized $\left(\mathrm{LP}_{1}\right)$ fixed at 0 degrees of the laboratory vertical and a Quarter-Waveplate $\left(\mathrm{QWP}_{1}\right)$, is placed. The $\mathrm{QWP}_{1}$ is inserted into a rotating platform that allows us to electronically control the orientation of its fast axis, generating at each different orientation a different incident State of Polarization (SoP).

The angle between the incident and the reflected beams is equal to 4 degrees and so, it can be assumed that the TNLCoS display is operating under quasi-normal incidence. In the light beam reflected by the TNLCoS display is set a Polarization State Detector (PSD), which is formed by Quarter-Waveplate $\left(\mathrm{QWP}_{2}\right)$ followed by a linear analyzer $\left(\mathrm{LP}_{2}\right)$. Different orientations of the $\mathrm{QWP}_{2}$ allow us to analyze different SoPs.

Finally, the radiometric measurements are performed by means of a commercial radiometer (Newport 1830-C) placed at the exit of the PSD. The combination of the PSD and the radiometer results in a complete Stokes polarimeter configuration (Chipman, 1995), which is capable of measuring any state of polarization exiting from the TNLCoS display.

The Mueller matrix $\mathrm{M}$ of a polarizing sample relates the incident and exiting (reflected, transmitted or scattered) states of polarization, described by the Stokes vectors $S_{\text {in }}$ and $S_{\text {ex }}$ respectively: 


$$
S_{\text {ex }}=M \cdot S_{\text {ex }}=\left(\begin{array}{llll}
m_{00} & m_{01} & m_{02} & m_{03} \\
m_{10} & m_{11} & m_{12} & m_{13} \\
m_{20} & m_{21} & m_{22} & m_{23} \\
m_{03} & m_{31} & m_{32} & m_{33}
\end{array}\right) S_{\text {in }}
$$

where $m_{j, k}(j=0,1,2,3$ and $k=0,1,2,3)$ are the coefficients of the Mueller matrix.

Due to the linear relation shown in Eq. (1), the Mueller matrix $M$ of a polarizing sample can be completely characterized by implementing a convenient independent linear equation system. In this way, the experimental LCoS display Mueller matrix is obtained by generating different incident SoPs (by means of the PSG in Fig. 1) and measuring their respective reflected ones by using different SoPs analyzers (implemented by means of the PSD in Fig. 1).

To completely characterize the $4 \times 4$ coefficients of the Mueller matrix describing the LCoS display, at least 4 independent incident SoPs and 4 independent analyzer SoPs must be used. Different sets of incident and analyzer SoPs are valid to this aim but the quality of the measurements will depend on different factors, such as the condition number (Taylor, 1974) of the matrices composed by the incident SoPs and the analyzer SoPs (De Martino et al., 2007; Peinado et al., 2010) or the number of SoPs selected (Peinado et al., 2010). Here, the calculation of the Mueller matrix is conducted as follows:

The intensity detected behind the PSD in Fig. 1 depends on the Stokes parameters of the light reflected by the LCoS display and on the orientation $\theta_{2}$ of the quarter-waveplate $\mathrm{QWP}_{2}$. It can be written as follows (Lizana et al., 2009):

$$
\mathrm{I}\left(\theta_{2}\right)=\frac{1}{2}\left[\mathrm{~S}_{0}+\mathrm{S}_{1} \cos ^{2}\left(2 \theta_{2}\right)+\mathrm{S}_{2} \sin \left(2 \theta_{2}\right) \cos \left(2 \theta_{2}\right)-\mathrm{S}_{3} \sin \left(2 \theta_{2}\right)\right]
$$

where $S_{0}, S_{1}, S_{2}$ and $S_{3}$ are the Stokes parameters of the light reflected from the LCoS display. As the intensity in Eq. (2) is a periodical signal with respect to the angle $\theta_{2}$, because it consists of different sinusoidal functions whose arguments are entire multiples of $\theta_{2}$, the method of synchronous detection (Goldstein, 2003) is applied. The synchronous detection represents an estimation of the coefficients of the Fourier series of this function.

By performing a summation of intensities corresponding to $\mathrm{N}$ different equally spaced values of $\theta_{2}$, completing a rotation of 360 degrees, some terms of Eq.(2) vanish due to the orthogonal properties of the sinusoidal sampled functions (Lizana et al., 2009). Besides, by performing another summation of different intensities, but now, multiplying the intensities by the sine or by the cosine of the corresponding angle $\theta_{2}$, different terms in Eq. (2) vanish. In this way, the following mathematical expression for the Stokes parameters is obtained (Lizana et al., 2009):

$$
\left(\begin{array}{c}
\mathrm{S}_{0} \\
\mathrm{~S}_{1} \\
\mathrm{~S}_{2} \\
\mathrm{~S}_{3}
\end{array}\right)=\left(\begin{array}{c}
2 \cdot \sum_{\mathrm{r}=1}^{\mathrm{N}} \mathrm{I}\left(\frac{\pi}{2}, \theta_{2, \mathrm{r}}\right)-4 \cdot \sum_{\mathrm{r}=1}^{\mathrm{N}} \mathrm{I}\left(\frac{\pi}{2}, \theta_{2, \mathrm{r}}\right) \cdot \cos \left(4 \theta_{2, \mathrm{r}}\right) \\
8 \cdot \sum_{\mathrm{r}=1}^{\mathrm{N}} \mathrm{I}\left(\frac{\pi}{2}, \theta_{2, \mathrm{r}}\right) \cdot \cos \left(4 \theta_{2, \mathrm{r}}\right) \\
8 \cdot \sum_{\mathrm{r}=1}^{\mathrm{N}} \mathrm{I}\left(\frac{\pi}{2}, \theta_{2, \mathrm{r}}\right) \cdot \sin \left(4 \theta_{2, \mathrm{r}}\right) \\
-4 \cdot \sum_{\mathrm{r}=1}^{\mathrm{N}} \mathrm{I}\left(\frac{\pi}{2}, \theta_{2, \mathrm{r}}\right) \cdot \sin \left(2 \theta_{2, \mathrm{r}}\right)
\end{array}\right)
$$

where $N$ is the number of selected angles $\theta_{2}$ and $\theta_{2, r}=\frac{2 \pi(r-1)}{N}$ with $r=1,2, \ldots, N$.

Once the SoP reflected by the LCoS display can be measured as described above, the next step is to fully characterize the LCoS display Mueller matrix. Next, the applied technique is 
described. The PSG in Fig. 1 is formed by the linear polarizer $\mathrm{LP}_{1}$, fixed at 0 degrees to the laboratory vertical, followed by the quarter-waveplate $\mathrm{QWP}_{1}$. The general expression for the states of polarization exiting from the PSG can be obtained by multiplying the Stokes vector of linearly polarized light in the horizontal direction (i.e. $\left.S_{\mathrm{P} 1}=(1,1,0,0)^{\mathrm{T}}\right)$ by the Mueller matrix of a linear retarder with a retardance equal to 90 degrees and whose orientation depends on the angle $\theta_{1}$ (Goldstein, 2003). We obtain the following Stokes vector:

$$
S_{\mathrm{PSG}}=\left(\begin{array}{cccc}
1 & 0 & 0 & 0 \\
0 & \cos ^{2} 2 \theta_{1} & \sin 2 \theta_{1} \cos 2 \theta_{1} & -\sin 2 \theta_{1} \\
0 & \sin 2 \theta_{1} \cos 2 \theta_{1} & \sin ^{2} 2 \theta_{1} & \cos 2 \theta_{1} \\
0 & \sin 2 \theta_{1} & -\cos 2 \theta_{1} & 0
\end{array}\right) \cdot\left(\begin{array}{l}
1 \\
1 \\
0 \\
0
\end{array}\right)=\left(\begin{array}{c}
1 \\
\cos ^{2} 2 \theta_{1} \\
\sin 2 \theta_{1} \cos 2 \theta_{1} \\
\sin 2 \theta_{1}
\end{array}\right)
$$

where different incident States of Polarization (SoPs) can be generated by varying the orientation angle $\theta_{1}$ of the $\mathrm{QWP}_{1}$. By replacing the Stokes vector given in Eq. (4) into Eq. (1), a general expression for the SoP exiting from the LCoS display is obtained:

$$
\mathrm{S}_{\mathrm{k}}\left(\theta_{1}\right)=\left(\mathrm{m}_{\mathrm{k} 0}+\frac{\mathrm{m}_{\mathrm{k} 1}}{2}\right)+\frac{\mathrm{m}_{\mathrm{k} 1}}{2} \cos 4 \theta_{1}+\frac{\mathrm{m}_{\mathrm{k} 2}}{2} \sin 4 \theta_{1}+\mathrm{m}_{\mathrm{k} 3} \sin 2 \theta_{1}
$$

where $k(k=0,1,2,3)$ indicates the different Stokes parameter and $m_{k, j}(j=0,1,2,3)$ are the different coefficients of the Mueller matrix.

Equation (5) comprises different sinusoidal functions whose arguments are entire multiples of $\theta_{1}$, and thus, the method of synchronous detection can be applied again. In particular, by performing a summation of different reflected SoPs corresponding to $\mathrm{N}$ different equally spaced values of $\theta_{1}$ we obtain the following expression:

$$
\sum_{\mathrm{r}=1}^{\mathrm{N}} \mathrm{S}_{\mathrm{k}}\left(\theta_{1, \mathrm{r}}\right)=\sum_{\mathrm{r}=1}^{\mathrm{N}}\left(\mathrm{m}_{\mathrm{k} 0}+\frac{\mathrm{m}_{\mathrm{k} 1}}{2}\right)+\sum_{\mathrm{r}=1}^{\mathrm{N}} \frac{\mathrm{m}_{\mathrm{k} 1}}{2} \cos 4 \theta_{1, \mathrm{r}}+\sum_{\mathrm{r}=1}^{\mathrm{N}} \frac{\mathrm{m}_{\mathrm{k} 2}}{2} \sin 4 \theta_{1, \mathrm{r}}+\sum_{\mathrm{r}=1}^{\mathrm{N}} \mathrm{m}_{\mathrm{k} 3} \sin 2 \theta_{1, \mathrm{r}}
$$

where $\mathrm{N}$ is the number of SoPs, $\mathrm{r}(\mathrm{r}=1,2, \ldots, \mathrm{N})$ indicates the specific term in the summation and with $\theta_{1, \mathrm{r}}=\frac{2 \pi(\mathrm{r}-1)}{\mathrm{N}}$.

By performing a summation of different reflected SoPs related to $\mathrm{N}$ different equally spaced values of $\theta_{1}$ on a complete rotation of 360 degrees, some terms of Eq. (6) vanish because of the orthogonal properties of the sinusoidal sampled functions (Lizana et al., 2009). Besides, by performing a summation of different reflected SoPs related to $\mathrm{N}$ different equally spaced values of $\theta_{1}$, but now, multiplying these SoPs by the sine or by the cosine of the corresponding angle $\theta_{1}$, different terms in Eq. (6) vanish. By doing this computation, an expression for the Mueller matrix of the LCoS display is obtained:

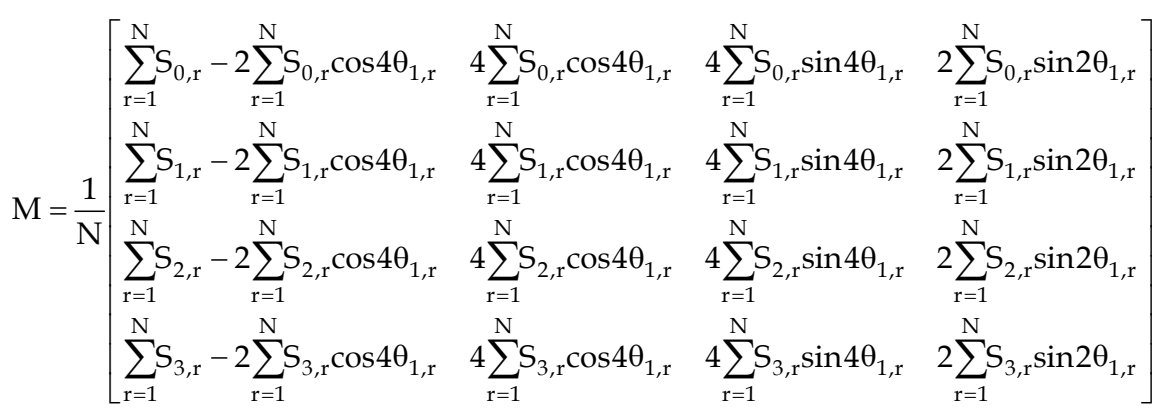


where $\mathrm{N}$ is the number of SoPs present in the summation, $\mathrm{r}(\mathrm{r}=1,2, \ldots, \mathrm{N})$ indicate the specific term of the summation and with $\theta_{1, \mathrm{r}}=\frac{2 \pi(\mathrm{r}-1)}{\mathrm{N}}$.

By experimentally measuring the quantities given in Eq. (7), the Mueller matrices of the Twisted Nematic LCoS display for different gray levels (from 0 to 255 in steps of 20 gray levels) have been obtained. The validity of the obtained results is tested by analyzing their capability to predict the SoP of the light reflected by the LCoS display.

As an example, in Fig. 2 we have plotted the Stokes parameters of the reflected SoP as a function of the gray level, when using an incident linear SoP at 135 degrees to the laboratory vertical. The spots represent the experimental data, which is measured by applying the SoP measuring method described above (see Eq. (2)). The continuous lines represent the theoretical values, which are calculated by multiplying the Stokes vector of a linear polarized light at 135 degrees (i.e. $\left.S_{135}=(1,0,-1,0)^{\mathrm{T}}\right)$ by the measured Mueller matrices of the TNLCoS display (see Eq. (1)). In all the cases, there is an excellent agreement between the predicted and the experimentally obtained Stokes parameters. Thus, the results given in Fig. 2 become an evidence of the effectiveness of the measured TNLCoS display Mueller matrices and validates the characterization method proposed.

It should be pointed out that depending on the specific application of the LCoS display, different physical parameters, such as the incident angle or the wavelength, can be required. In such situations, the LCoS display response can be notably different. However, the characterizing method described in this section can be extended to different experimental conditions, such as the incident angle (Lizana et al., 2009) or the wavelength (Lizana et al., 2008c).

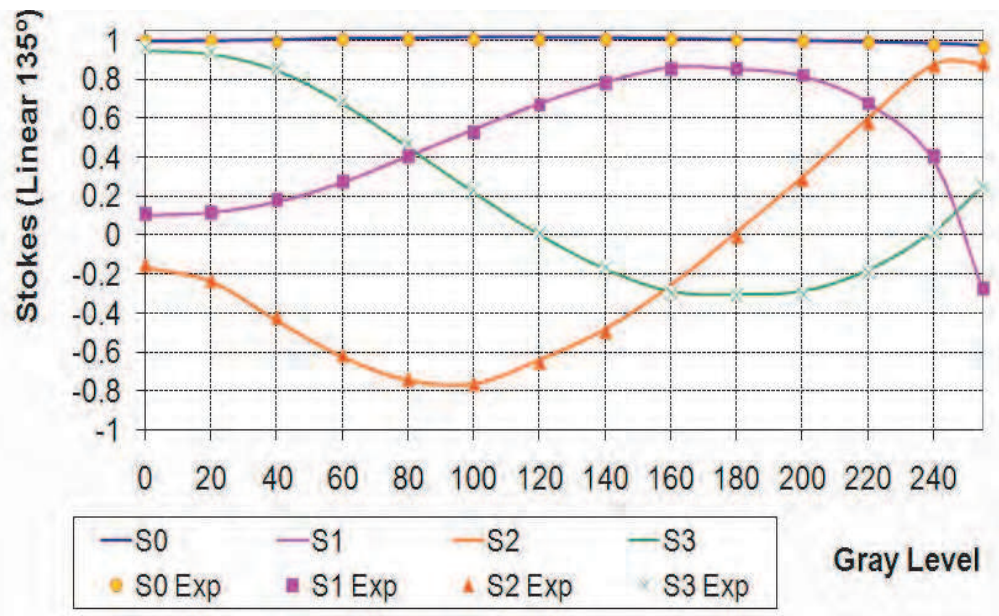

Fig. 2. Stokes parameters as a function of the gray level for an incident SoP linear at 135 degrees of the laboratory vertical. Whereas the spots represent the experimental data, the continuous lines represent the theoretical values.

\subsection{Mueller to Jones conversion: Phase prediction}

In this subsection, a methodology useful to predict the phase response of LCoS displays is proposed. To this aim, since Jones matrices contains the required phase information, a 
conversion of the experimentally obtained LCoS display Mueller matrices to Jones matrices is applied.

As stated above, LCoS displays introduce a certain amount of unpolarized light in the reflected beam (Lizana et al., 2008a; Márquez et al., 2008; Wolfe \& Chipman, 2006). Since Jones matrices do not describe unpolarized samples, a direct conversion from the experimental Mueller matrices to the Jones matrices can not be directly performed. In this situation, a decomposition of the LCoS display Mueller matrices into different basic Mueller matrices becomes very helpful. There exist different ways to decompose depolarizing Mueller matrices (Ossikovski et al., 2008). All these different decompositions provide significant polarimetric information and they become more or less practical as a function of the specific application for which they are required.

Here, the Lu-Chipman decomposition (Goldstein, 2003), which is a natural generalization of the polar decomposition (Lu \& Chipman, 1996) to the depolarizing case, is applied. The LuChipman decomposition states that a general Mueller matrix can be decomposed as the product of three basic Mueller matrices: the Mueller matrix of a pure diattenuator $M_{D}$ (an optical element that changes the orthogonal amplitudes unequally), the Mueller matrix of a pure retarder $\mathrm{M}_{\mathrm{R}}$ (an optical element that introduces a phase-shift between the orthogonal components), and the Mueller matrix of a depolarizer $\mathrm{M}_{\Delta}$ (an optical element that introduces certain amount of unpolarized light). In the case of LCoS displays, diverse polarimetric studies have proved that they are non-diattenuating devices (Lizana et. al, 2008c; Lizana et al., 2009; Márquez et al., 2008) and the corresponding $M_{D}$ matrices can be approximated to the identity matrix. Therefore, when applying the Lu-Chipman polar decomposition to the LCoS display case, the following relation holds (Moreno et al., 2008):

$$
\mathrm{M}_{\mathrm{LCoS}}=\mathrm{M}_{\Delta} \mathrm{M}_{\mathrm{R}}=\left(\begin{array}{cc}
1 & \overrightarrow{0}^{\mathrm{T}} \\
\overrightarrow{\mathrm{P}}_{\Delta} & \mathrm{m}_{\Delta}
\end{array}\right) \cdot\left(\begin{array}{cc}
1 & \overrightarrow{0}^{\mathrm{T}} \\
\overrightarrow{0} & \mathrm{~m}_{\mathrm{R}}
\end{array}\right)
$$

where $\overrightarrow{0}^{\mathrm{T}}=(0,0,0,0), \overrightarrow{\mathrm{P}}_{\Delta}$ refers to the polarizance vector (Goldstein, 2003), and the matrices denoted as $m_{\Delta}$ and $m_{R}$ are $3 \times 3$ submatrices whose coefficients can be calculated by following the procedure described in (Goldstein, 2003).

The retarder component $\mathrm{M}_{\mathrm{R}}$ of the Mueller matrix given in Eq. (8) is a non-absorbing, unitary and fully polarized Mueller matrix. Under these conditions, the equivalent Jones matrix of the retarder can be obtained from the $M_{R}$ component (Moreno et al., 2008). It can be done by following the relationships between the Mueller matrix coefficients and the Jones matrix coefficients given in Ref. (Goldstein, 2003).

Once the equivalent Jones matrices of the TNLCoS are calculated, a methodology for the evaluation of the complex phase modulation is applied. We follow the technique proposed in Ref. (Moreno et al., 2003), being suitable to predict the phase response in LCDs. This technique considers that any non-absorbing reciprocal polarization device can be described by an unimodular unitary Jones matrix (Fernández-Pousa et al., 2000), the TNLCoS displays being a particular case. Then, such devices can be described by the following relation:

$$
\mathrm{J}_{\mathrm{LCoS}}=\mathrm{e}^{-\mathrm{i} \beta}\left(\begin{array}{cc}
\mathrm{A} & \mathrm{B} \\
-\mathrm{B}^{*} & \mathrm{~A}^{*}
\end{array}\right)
$$

where $A=A_{R}-i A_{I}$ and $B=B_{R}-i B_{I}$ are complex parameters (subscripts $R$ and $I$ indicate the real and imaginary parts) which depend on the applied voltage $\mathrm{V}$ and fulfill the condition $|A|^{2}+|B|^{2}=A_{R}^{2}+A_{I}^{2}+B_{R}^{2}+B_{I}^{2}=1$. 
From the calculated matrices $M_{R}$ and the transformations given in (Goldstein, 2003), the complex parameters A and B are fully calibrated as a function of the gray level. However, no information about the external phase $\beta$ in Eq. (9) is obtained. The knowledge of the external phase $\beta$ is critical to accurately predict the phase modulation of LCoS displays and so, the application of a technique to determine its value becomes mandatory.

A general expression for the total phase $\delta$ of a LCD inserted between two polarizers (whose orientations are $\theta_{1}$ and $\theta_{2}$ respectively) is provided in Ref. (Moreno et al., 2003):

$$
\delta=\beta+\delta_{M}=\beta+\operatorname{atan}\left(\frac{A_{I} \cos \left(\theta_{1}+\theta_{2}\right)+B_{I} \sin \left(\theta_{1}+\theta_{2}\right)}{A_{R} \cos \left(\theta_{1}-\theta_{2}\right)+B_{R} \sin \left(\theta_{1}-\theta_{2}\right)}\right)
$$

where $\delta_{\mathrm{M}}$ is a phase contribution that depends on the polarizer orientations $\theta_{1}$ and $\theta_{2}$.

As the coefficients $A_{R}, A_{I}, B_{R}$ and $B_{I}$ are known, the phase $\delta_{M}$ in Eq. (10) can be calculated for a given pair of orientations $\theta_{1}$ and $\theta_{2}$. Besides, the total phase $\delta$ as a function of the gray level can be experimentally determined, for instance, by using the interferometric based set-up given in Ref. (Lizana et al., 2008b). In this situation, the external phase $\beta$ is readily obtained by isolating it from Eq. (10).

Since the external phase $\beta$ does not depend on the incident and analyzer SoPs, once it is measured, the phase response of LCoS display as a function of the gray level can be completely predicted.

\subsection{LCoS display optimizing procedure}

In digital holography applications, the diffraction efficiency is maximized when operating in the phase-only modulation regime (Moreno et al., 2004). Therefore, in such applications is very desirable to operate with an LCoS display response achieving a linear phase modulation up to 360 degrees, without coupled depolarization or coupled amplitude modulation.

By following the methods given in subsections 2.1 and 2.2, we are able to predict the intensity and the phase response of LCoS display. By taking advantage of this prediction capability, an optimization procedure can be devised to maximize the efficiency of digital holograms generated with LCoS displays.

Here, we apply an optimizing method, based on a numerical search algorithm, which allows us to achieve pairs of incident and analyzer SoPs that provide an LCoS display working in the phase-only regime. By starting from an initial pair of incident and analyzer SoPs, the numerical search algorithm varies these parameters in order to maximize the figure of merit QPO shown below. The SoP searching process enables to use any type of fully polarized light (i.e. linearly polarized light, circularly polarized light and elliptically polarized light). In this way, better results than those obtained when using only linear polarized light are achieved (Márquez et al., 2008).

The figure of merit $\mathrm{Q}_{\mathrm{PO}}$ is based on a specific criterion to evaluate the quality of the response in the phase-only regime. In particular, it is based on three conditions: minimum transmittance value as high as possible, maximum phase modulation and minimum transmittance contrast.

$$
\mathrm{Q}_{\mathrm{PO}}=\frac{1}{\lambda_{1}+\lambda_{2}+\lambda_{3}}\left[\lambda_{1} \frac{\Delta \mathrm{\tau}}{2 \pi}+\lambda_{2}(1-\Delta \mathrm{T})+\lambda_{3} \mathrm{~T}_{\mathrm{m}}\right]
$$

where $\Delta \tau$ is the maximum phase difference, $\Delta \mathrm{T}$ is the maximum transmittance difference and $\mathrm{T}_{\mathrm{m}}$ denotes the minimum transmission value. Moreover, the parameters $\lambda_{1}, \lambda_{2}$ and $\lambda_{3}$ are 
coefficients enabling us to vary the specific weight of the three parameters involved into the optimization process.

By maximizing the figure of merit given in Eq. (11), different TNLCoS display configurations have been obtained, providing excellent results for phase-only response (Márquez et al., 2008), as for instance, those shown in Fig. 3.

In Fig. 3, the intensity values (left axis) and the phase values (right axis) are represented as a function of the gray level, the former being plotted in pink and the latter plotted in brown. Whereas the predicted values are represented by curves, the measured experimental data are represented by spots. For the measurement of the phase (brown squares), the interferometer based set-up given in Ref. (Lizana et al., 2008b) has been applied. The experimental orientations of external waveplates (WP) and linear polarizers (LP) used to achieve the incident and analyzer SoPs for this optimized configuration are: incident $\left(\mathrm{LP}_{1}=45\right.$ degrees, $\mathrm{WP}_{1}=160$ degrees $)$; analyzer $\left(\mathrm{LP}_{2}=-41\right.$ degrees, $\mathrm{WP}_{2}=18$ degrees $)$.

We see an excellent agreement between experimental and theoretical values, validating the suitability of the employed optimization methodology. Besides, good results for phase-only modulation are achieved. In fact, an almost constant intensity curve as a function of the gray level, with a phase-shift approximately of 300 degrees is obtained.

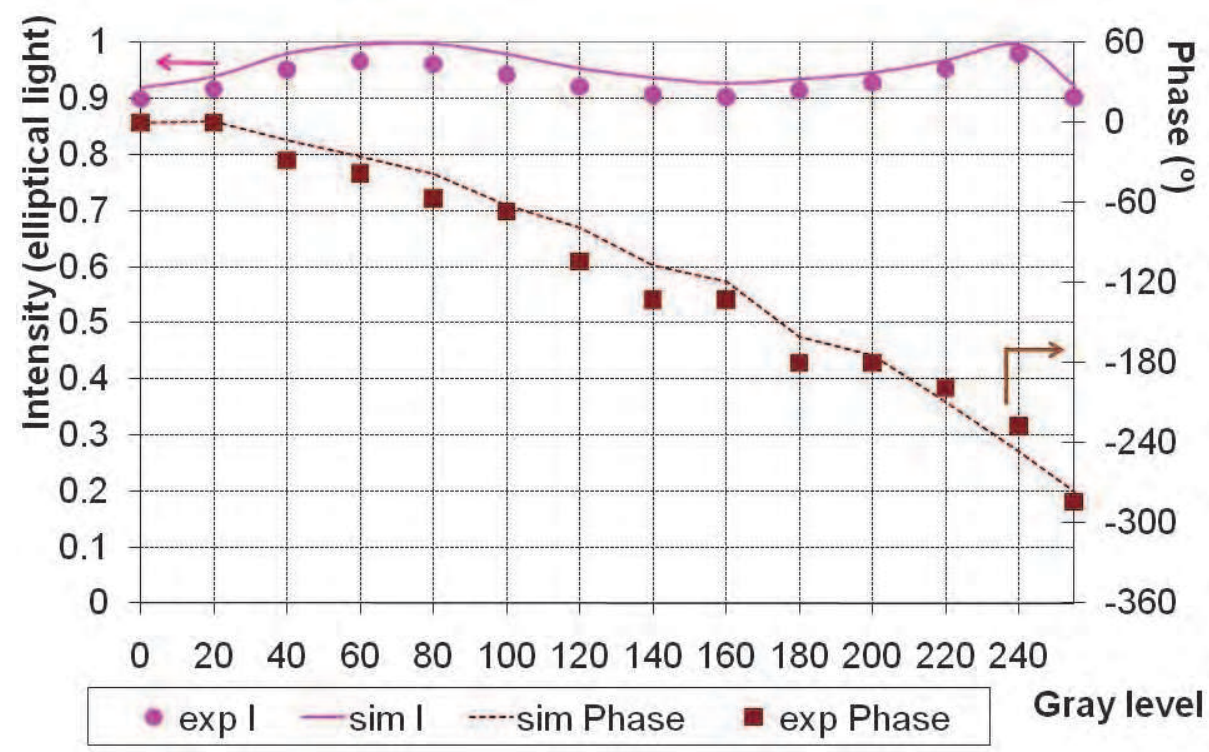

Fig. 3. TNLCoS display phase only response as a function of the gray level.

Finally, we have allowed the numerical search procedure to decrease the minimum value of transmittance $\mathrm{T}_{\mathrm{m}}$, in order to reach a higher phase-shift. The obtained result is shown in Fig. 4. The experimental orientations of external waveplates (WP) and linear polarizers (LP) are the following: incident beam $\left(\mathrm{LP}_{1}=88\right.$ degrees, $\mathrm{WP}_{1}=7$ degrees); analyzer $\left(\mathrm{LP}_{2}=90\right.$ degrees, $\mathrm{WP}_{2}=-15$ degrees).

In Fig. 4, we see an excellent result in terms of phase-only regime. In particular, a continuous intensity response as a function of the gray level, which keeps almost constant 
around the value 0.65 , is achieved. Besides, it is accompanied by a very large phase-shift, close to 360 degrees.

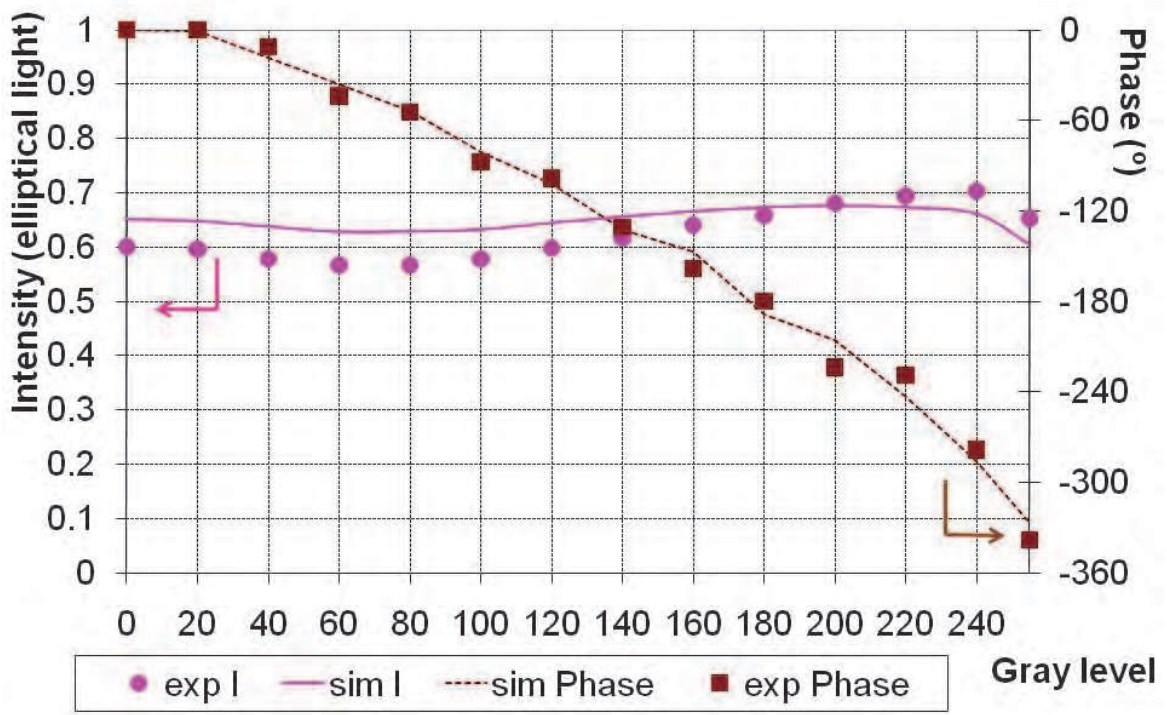

Fig. 4. TNLCoS display phase only response as a function of the gray level.

\section{Time-fluctuations of the phase: Effects on the digital hologram efficiency}

Experimental evidence of the time-fluctuations of the phase phenomenon, observed when working with LCoS displays (Lizana et al., 2008b; Lizana et al., 2010), are provided in this section. This phenomenon originates from the electrical addressing schemes (Hermerschmidt et al., 2007) applied in LCoS displays, and it may notably degrade the performance of LCoS displays when generating digital holograms (Lizana et al., 2008b). To provide greater insight into this topic, this section also includes a study of the timefluctuations of the phase effects on the efficiency of diverse basic digital holograms generated with the TNLCoS display.

\subsection{Time-fluctuations of the phase: Experimental evidence}

To experimentally measure the time-fluctuations of the phase phenomenon, we have applied the diffraction based set-up sketched in Fig. 5.

We employ an unexpanded beam of a He-Ne laser $(633 \mathrm{~nm})$ to illuminate the TNLCoS display, with an angle of incidence equal to 2 degrees. A Polarization State Generator (PSG) is placed in the incident beam, composed of a Linear Polarizer $\left(\mathrm{LP}_{1}\right)$ and a Quarter Wave Plate $\left(\mathrm{QWP}_{1}\right)$, and a Half Wave Plate (HWP) is introduced in front of the PSG, allowing us to control the intensity of light incoming to the PSG. In the reflected beam, we have placed a Polarization State Detector (PSD), composed of a Quarter Wave Plate $\left(\mathrm{QWP}_{2}\right)$ followed by a Linear Polarizer $\left(\mathrm{LP}_{2}\right)$. We selected the PSG and PSD configuration in Fig. 5, which yields phase-only modulation with constant transmittance (Márquez et al., 2008). Then, we address 
a binary grating to the TNLCoS display, and by means of two radiometers (Newport 1830C) placed in the far diffraction plane, the intensities of the zero and first diffracted orders are captured. The signals detected by the radiometers are synchronized and displayed on an oscilloscope (Tektronix TDS3012B), allowing us to perform intensity measurements of the diffracted orders as a function of the time.

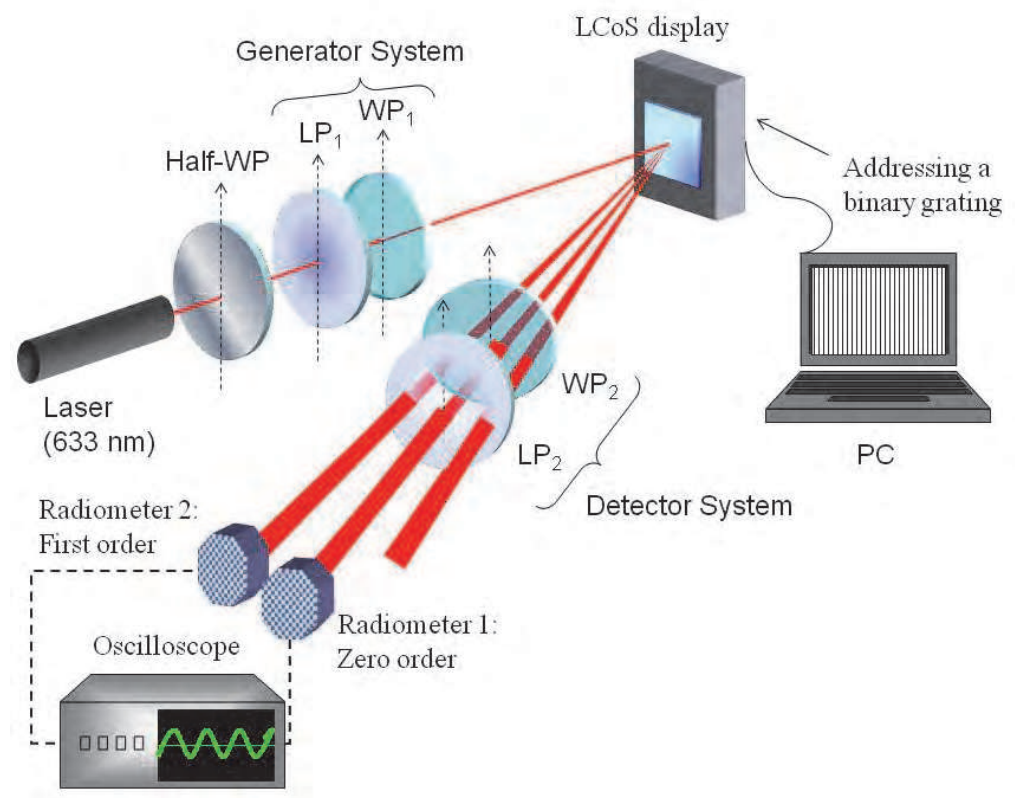

Fig. 5. Diffraction based set-up to measure the phase modulation as a function of the time.

Using the set-up sketched in Fig. 5, the intensity at the zero and at the first diffracted orders was measured as a function of the time, when addressing three different binary gratings. In particular, three binary gratings with a different pair of gray levels are used: $(0,120),(0,211)$ and $(0,255)$. The obtained results are given in Fig. 6 . Whereas the intensity at the zero order is plotted in black, the intensity at the first order is plotted in red. The intensity values in Fig. 6 are normalized to the mean value of the intensity measured at the zero order, when a constant image is addressed to the LCoS display with the reference gray level (i.e. zero gray level).

Whereas in Fig. 6 (a) and in Fig. 6 (c), the zero order is in general more intense than the first diffraction order, in Fig. 6(b), the first order intensity is greater than the zero order intensity. This occurs because by changing the addressed gray level pair, the phase difference between the two parts of the binary grating varies. Thus, the quantity of light in each diffracted order in the far diffraction plane varies as well. However, the intensity of the diffraction orders is periodically fluctuating as a function of the time in all cases. This fact points out the existence of time-fluctuations of the phase phenomenon.

A well-known digital hologram is the binary grating with a phase difference of 180 degrees. Theoretically, when addressing such a binary grating, a null zero order has to be obtained. In addition, it has to be accompanied by the \pm 1 diffractive orders, whose intensity values 
should posses approximately the $40 \%$ of the input light. Therefore, the results obtained in Fig. $6(\mathrm{~b})$ correspond in some instants of time to a binary grating with a phase difference equal to 180 degrees. In fact, in some intervals of time, the maximum value of the intensity at the first diffraction order reaches 0.4 and the zero order vanishes.
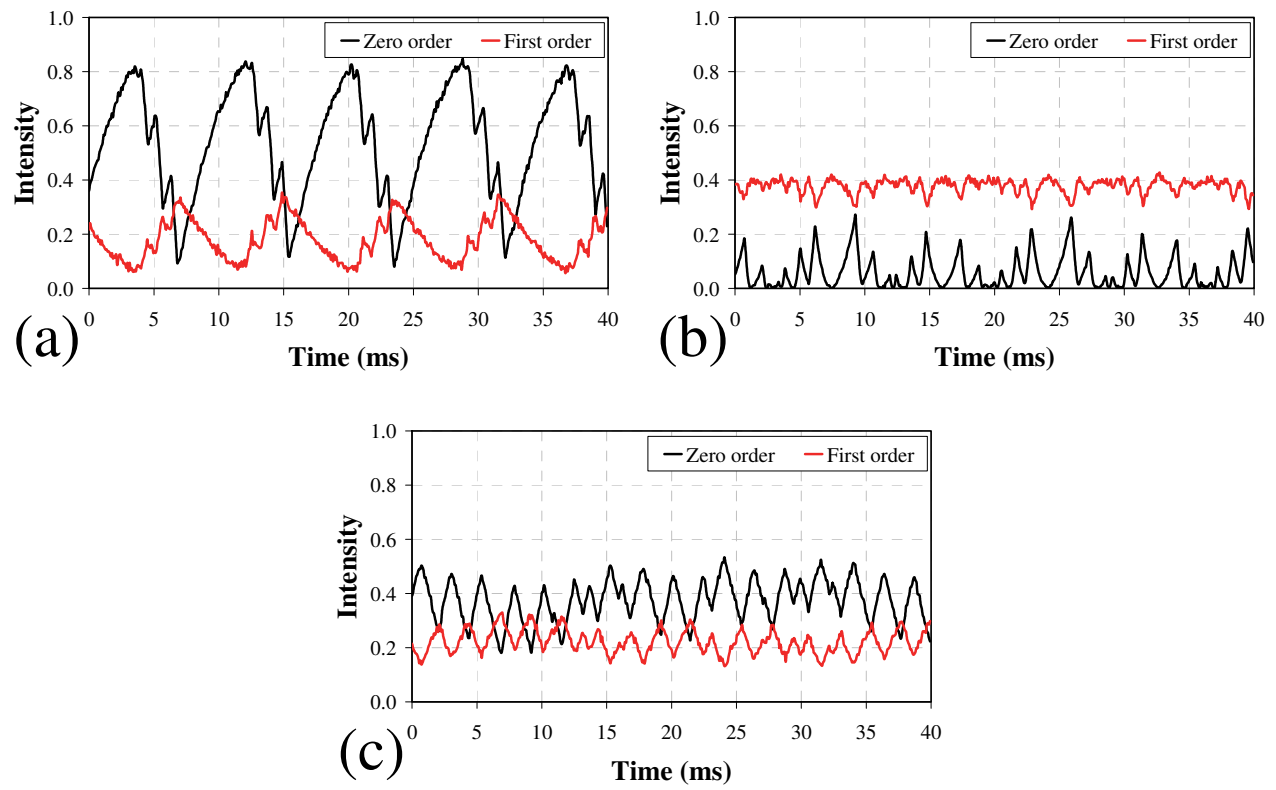

Fig. 6. Intensity at the zero (in black) and at the first (in red) diffractive orders, when addressing a binary grating containing the gray levels: (a) 0-120, (b) 0-211 and (c) 0-255.

As the TNLCoS display is working in the phase-only regime, the two gray levels applied for the binary gratings addressed to the TNLCoS display present the same values for the amplitude but a phase difference $\Phi(t)$ which depends on the time. At this point, an analytical expression for the intensities at the zero and the first diffracted orders can be derived as a function of $\Phi$ (Zhang et al., 1994). In particular, when the periodic structure of the grating is formed by two levels of the same size (i.e. a duty cycle of $50 \%$ ), the normalized intensities as a function of the time, in the zero order and in the \pm 1 orders, can be described as follows:

$$
\begin{array}{r}
\mathrm{I}_{0}(\mathrm{t})=\frac{1}{2}(1+\cos \Phi(\mathrm{t})) \\
\mathrm{I}_{ \pm 1}(\mathrm{t})=\frac{2}{\Pi^{2}}(1-\cos \Phi(\mathrm{t}))
\end{array}
$$

From the relations given in Eq. (12) and (13), an expression for the phase modulation as a function of the time can be deduced:

$$
\cos (\Phi(t))=\frac{4 I_{0}(t)-\Pi^{2} I_{1}(t)}{4 I_{0}(t)+\Pi^{2} I_{1}(t)}
$$


From the obtained intensity values in Fig. 6, the phase $\Phi(\mathrm{t})$ as a function of the time can be calculated by applying Eq. (14). The results corresponding to the three binary gratings applied above (i.e. gray levels $(0,120),(0,211)$ and $(0,255))$ are given in Fig. 7 .

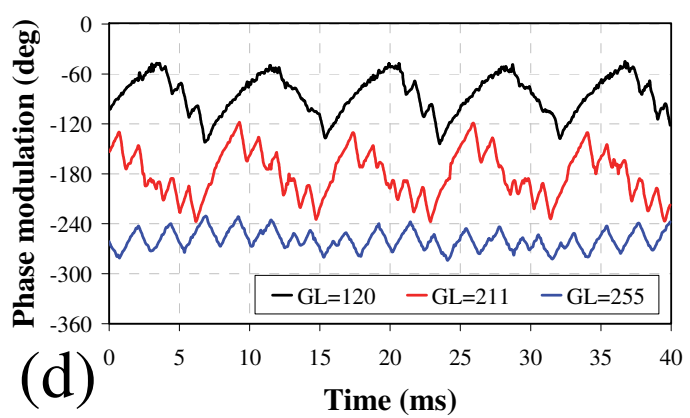

Fig. 7. Time fluctuations of the phase when addressing a binary grating containing the gray levels: 0-120 (in black), 0-211 (in red) and 0-255 (in blue).

The results shown in Fig. 7 constitute an experimental evidence for the time-fluctuations of the phase phenomenon, whose fluctuation amplitude depends on the gray levels applied to the binary gratings addressed. Note that the phase fluctuations can reach very high values, as in the case of the grating $(0,211)$, where the mean phase difference is equal to 180 degrees, but it is accompanied with a great fluctuation amplitude of almost 120 degrees (Fig. 7, in red). As proved in this subsection, the phase-shift measured at the TNLCoS reflected beam is far from being constant along the frame period, leading to undesired effects that should be evaluated depending on the application.

\subsection{Time-fluctuations of the phase: Effects on the digital holograms efficiency}

In this section, we analyze the effect of time-fluctuations of the phase on the efficiency of two basic digital holograms, generated with the TNLCoS display. Both digital holograms have an average phase shift of 180 degrees.

The TNLCoS display has been configured to work in the phase-only regime by using the configuration related to Fig. 4 . Then, by means of the combination of a spatial filter and a convergent lens, the whole TNLCoS display area has been illuminated with a collimated beam. The reflected image is captured with a CCD camera placed in the back focal plane of a second convergent lens set in the reflected beam. In this way, the CCD camera is able to capture the Fourier transform spectrum obtained by diffraction.

First, we address a binary phase-only grating with an average phase shift of 180 degrees to the TNLCoS display. The image captured with the CCD camera is shown in Fig. 8, where the typical diffraction orders generated by the binary grating are observed.

Although the intensity related to the \pm 1 diffracted orders is stronger (brighter spots) than the one captured at the zero diffracted order, the later does not completely vanish, as would be expected in a binary grating with a phase difference of 180 degrees. 


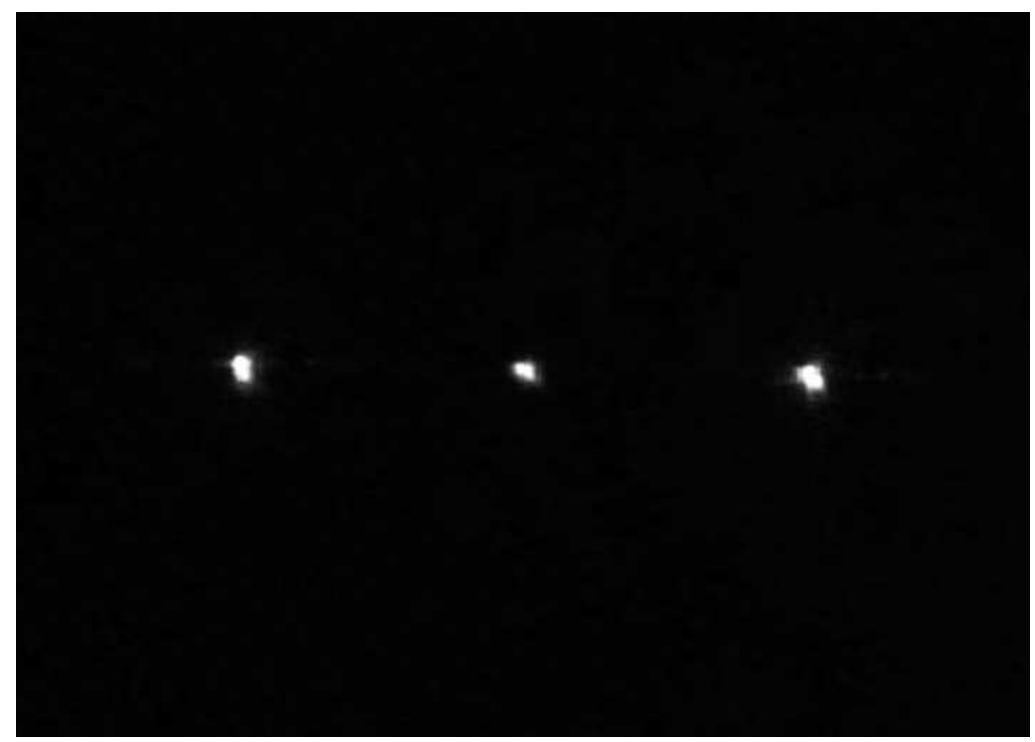

Fig. 8. Diffraction pattern captured with a CCD camera when addressing a binary grating with an averaged phase difference of 180 degrees.

This fact can be understood by comparing the results shown in Fig. 8 with those given in Fig. 6(b). As seen in Fig. 6(b), in those instants of time where the zero diffraction order is exactly equal to zero, the intensity of the first diffracted order is about 0.4 . In such instants of time, the results are consistent with a binary phase grating with an instantaneous phase difference equal to 180 degrees. However, the intensity of the diffraction orders detected with the CCD camera (Fig. 8) corresponds to the mean values of the intensity measurements as a function of the time given in Fig. 6 (b). Therefore, as the intensity at the zero and at the \pm 1 diffracted orders varies as a function of the time (because the phase is fluctuating), the mean zero order is higher than 0 and the mean \pm 1 diffracted orders are lower than 0.4 (in particular, 0.059 for the zero order and 0.377 for the \pm 1 orders).

Next, we have displayed a binary phase computer generated hologram designed to reconstruct a butterfly. Again, the averaged phase-shift between gray levels is equal to 180 degrees. The results are shown in Fig. 9. We have added a linear phase along the diagonal direction with the aim of spatially separating the reconstructions of the different orders (i.e. the zero and \pm 1 orders are separated in this way). The butterfly is reconstructed at the \pm 1 orders, with an efficiency equivalent to those obtained in the first diffraction order in Fig. 8 . The reconstructed butterflies are accompanied by a zero order diffracted with non-zero intensity that we can see as a bright peak located at the optical axis. As in the binary grating case, the intensity value measured at the zero diffracted order originates from timefluctuations in the phase modulation. 


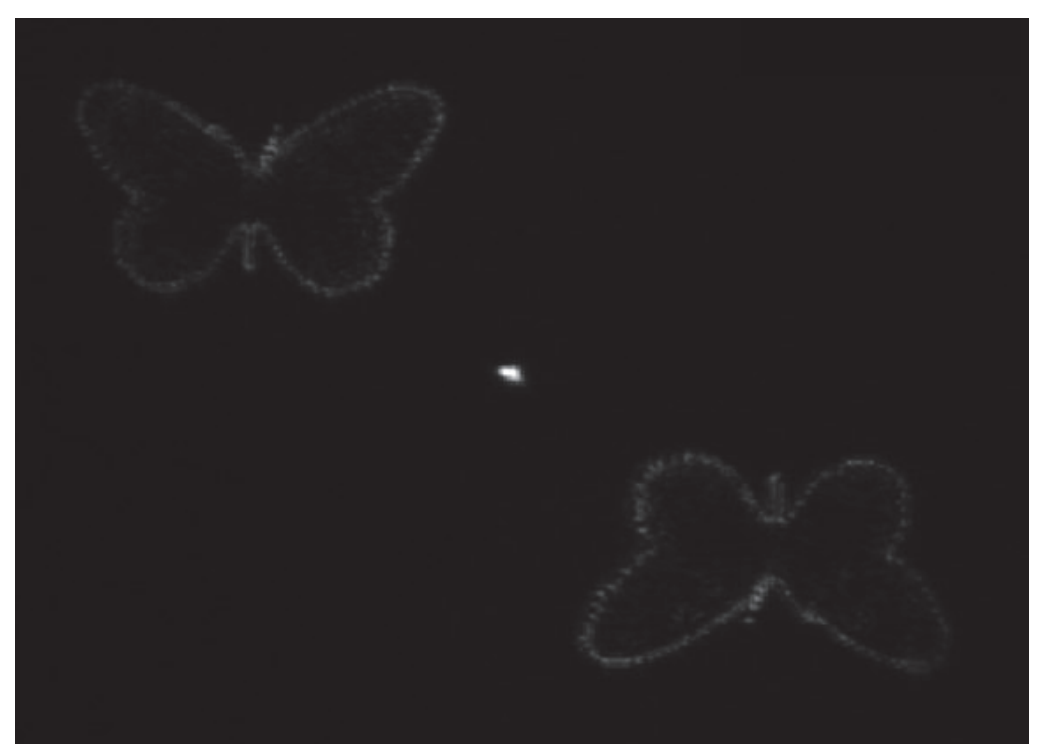

Fig. 9. Digital hologram designed to reconstruct a butterfly.

\section{Enhancement of the digital hologram efficiency in the presence of time- fluctuations of the phase by applying the Minimum Euclidean principle}

In this section, another type of LCoS display, the Parallel Aligned (PA) LCoS display model, is studied for use in the generation of digital holograms. The PALCoS display prototype under analysis is a commercial electrically controlled birefringence LCoS display distributed by HoloEye systems. This prototype is a PLUTO Spatial Light Modulator (SLM) with a diagonal display of $1.8 \mathrm{~cm}$. The pixel pitch is of $8 \mu \mathrm{m}$ with a fill factor of $87 \%$ and the display has a resolution of $1920 \times 1080$. Besides, this LCoS display model allows us to upload different electrical sequences formats for the digital electrical addressing, which are based on different pulsed-width modulation schemes. The use of different electrical sequences may result in different responses and efficiencies of the PALCoS display (Hermerschmidt et al., 2007; Moore et al., 2008).

A characteristic feature in PALCoS displays is that the LC molecules in such devices are all parallel aligned. Then, by illuminating the display with an incident linear State of Polarization (SoP) parallel to the LC extraordinary axis, the exiting SoP is constant as a function of the time, even in the presence of LC fluctuations. In this situation, the PALCoS display is working in the phase-only regime and the effective depolarization phenomenon can be avoided. However, the damaging effect of the time-fluctuations of the phase phenomenon described in section 3 is still present.

Here, the significance of the time-fluctuations phenomenon in our PALCoS display is experimentally analyzed for the available electrical sequences. Moreover, two different 
possible mapping schemes for the ideal phase-only function implementation of digital holograms are considered: a linear phase mismatching scheme and a mapping scheme based on the minimum Euclidean distance principle (Juday, 2001; Moreno et al., 1995). They are experimentally tested in the presence of time-fluctuations of the phase, in order to find the best configuration to maximize the efficiency of digital holograms generated with the PALCoS display (Lizana et al., 2010).

\subsection{Time-fluctuations of the phase in the PALCoS display}

To operate in the phase-only regime, the PALCoS display under analysis has been sandwiched between two polarizers oriented in the direction of the LC extraordinary axis. By using the diffraction based set-up shown in Fig. 5, we have measured the phase response as a function of the time for three different digital addressing sequences provided by HoloEye (since now labelled as the sequences \#1, \#2 and \#3). For each sequence employed, the phase as a function of the time is measured for different gray levels. In all the cases, the measurements are made by using an unexpanded He-Ne laser beam $(633 \mathrm{~nm})$ and at quasinormal incidence (i.e. an incident angle of 2 degrees).

Figure 10 shows the PALCoS phase response as a function of the time for the addressing sequences \#1 (Fig. 10(a)), \#2 (Fig. 10(b)) and \#3 (Fig. 10(c)).
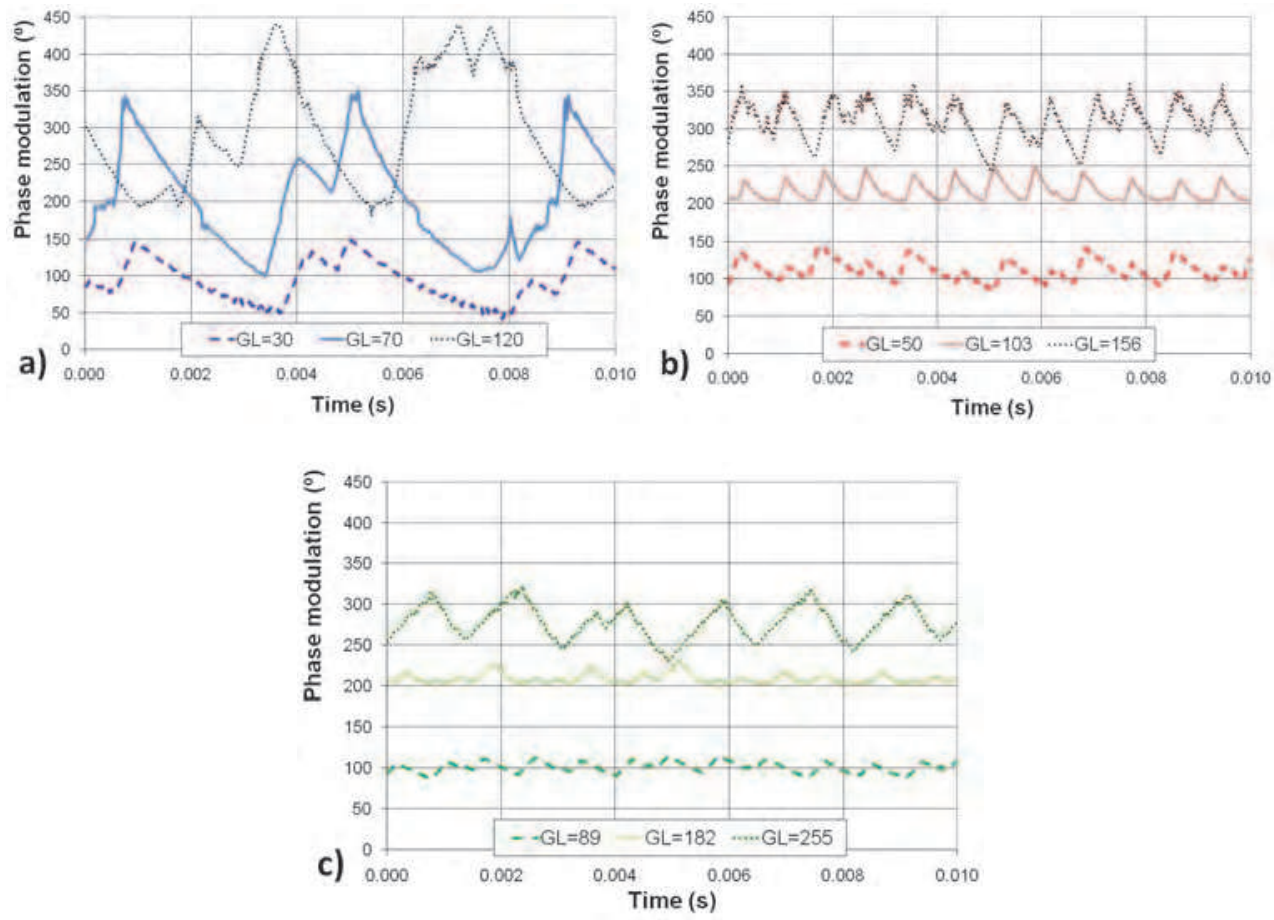

Fig. 10. Phase modulation as a function of time for different gray levels, for the addressing sequences: (a) \#1, (b) \#2 and (c) \#3. 
In every case, the measurements are done for three different gray levels, which lead to average phase values of 100 degrees, 200 degrees and 300 degrees respectively. In this way, we can assume that an estimation of the whole phase domain is conducted.

Figure 10 shows that for all the addressing sequences, periodic time-fluctuations of the phase are obtained. Besides, the amplitude of the phase fluctuations depends on the addressed sequence. In particular, whereas the fluctuations for the sequence \#1 (Fig. 10(a)) are very large, they become much smaller for the sequences \#2 and \#3 (Fig. 10(b) and Fig. 10 (c) respectively).

To generate digital holograms with LCoS displays, the required phase distribution has to be implemented. To this aim, the significant parameter is the mean phase. Thus, the mean phase modulation curves for the addressed sequences \#1, \#2 and \#3 are measured as well. The mean phase values have been obtained by calculating the difference between the eigenvalues of the PALCoS display equivalent Jones matrices, which are obtained as described in subsection 2.2.

The results are shown in Fig. 11, where the phase modulation for the sequences \#1 (in blue) and \#2 (in red) are larger than 360 degrees (i.e. 520 degrees and 480 degrees). On the other hand, the phase depth for the sequence \#3 is clearly smaller (about 280 degrees).

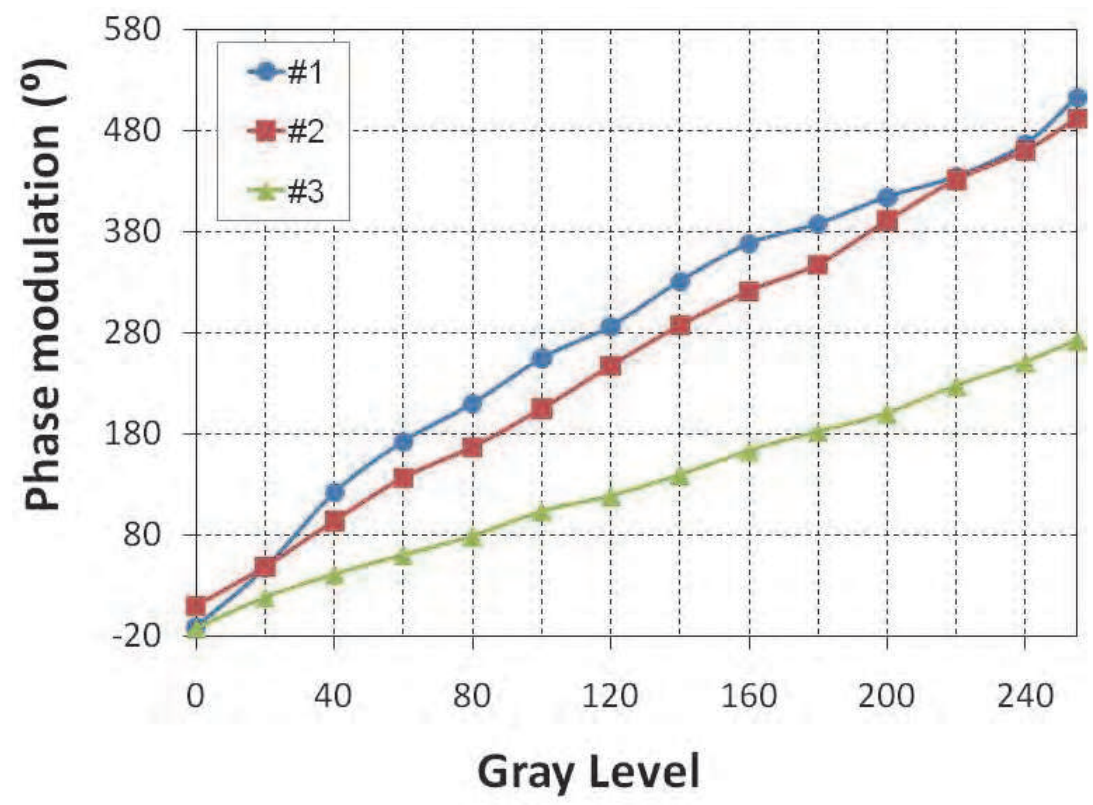

Fig. 11. Phase modulation as a function of the gray level for the sequence \#1 (in blue), \#2 (in red) and \#3 (in green).

\subsection{Digital hologram efficiency evaluation: Application of the minimum Euclidean distance principle}

Figure 11 shows how the different electrical sequences available with the PALCoS display provide different phase modulation depths. In general, phase modulations larger than 360 
degrees are desired to maximize the efficiency of holograms generated with LCDs. However, as shown in Fig. 10, the electrical sequences applied in LCoS display produce time-fluctuations of the phase, which degrade the efficiency of the generated holograms. Thus, small amplitude of the fluctuations is desired as well.

In this situation, to maximize the efficiency of digital holograms generated with LCoS displays, a trade-off between phase modulation depth (as large as possible) and amplitude of the time-fluctuations of the phase (as small as possible) has to be found.

Next, the suitability of the different electrical sequences to maximize the efficiency of the implemented holograms is experimentally analyzed. Besides, different mapping schemes for phase-only distribution implementation are also reviewed.

\subsubsection{Linear phase mismatching and saturated mismatching encoding schemes}

To accurately implement digital holograms with LCDs, it is very important to experimentally generate a real phase distribution as close as possible to the designed one. However, as a consequence of diverse non-linearities related to the experimental implementation, the ideal phase distribution is never achieved. To reduce different LCDs degradation sources, some authors have proposed diverse strategies (Davis et al., 1998; Márquez et al., 2001). For instance, if the available dynamic range is less than 360 degrees, the minimum Euclidean distance projection principle (Juday, 2001; Moreno et al., 1995) can be applied. In this way, the modulation diffraction efficiency may be greatly enhanced by selecting an appropriate mapping scheme for the implementation of the phase function onto the restricted phase-only domain (Moreno et al., 1995).

Next, two possible mapping schemes, for the ideal phase-only distribution implementation of holograms, are reviewed. These schemes are represented in Fig. 12, where the truly

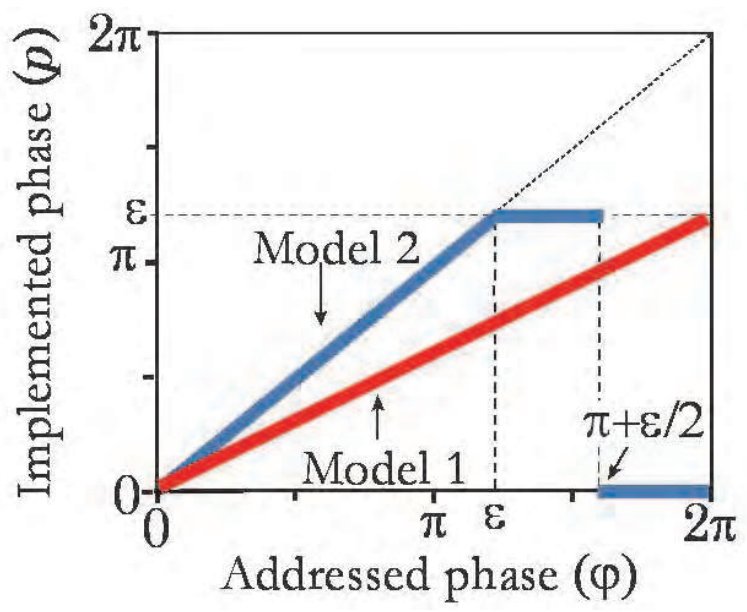

Fig. 12. Mapping scheme implementation: In red, the linear mismatching encoding (model 1) and in blue, the saturation mismatching encoding (model 2). 
implemented phase (denoted as $p$ ) is represented as a function of the addressed phase (denoted as $\varphi$ ). Note that the diagonal dotted line represents the correct matching between the designed phase $\varphi$ and the displayed phase $p$.

We assume that the phase values available with the applied LCD are in the range $(0, \varepsilon)$, with $\varepsilon<2 \pi$. First, the red line (model scheme 1 ) represents a linear phase mismatching. Second, the blue line (model scheme 2) represents a more efficient encoding scheme, which we denote as saturated mismatching encoding. Model 2 represents the perfect phase matching up to the maximum modulation depth $\varphi=\varepsilon$, while there is a saturation for values $\varphi>\varepsilon$. Then, for each phase $\varphi>\varepsilon$, the closest available phase value in the modulation domain is taken by following the minimum Euclidean distance principle (see Fig. 12, in blue).

\subsubsection{Experimental results}

In this subsection, we compare the efficiency of a basic continuous digital hologram, the blazed grating (Fujita et al., 1982), generated with our PALCoS display when uploading the different electrical sequences (i.e. sequences \#1, \#2 and \#3). For all the sequences, the implementation is conducted by using the linear phase mismatching scheme. In this way, we have limited the phase range between 0 and 360 degrees and we have applied a look-up-table to produce a linear increment for the average phase values. Besides, the phase modulation provided by sequence \#3 is lower than 360 degrees (see Fig. 11), and so, the saturated mismatching encoding is applied for this sequence as well.

In all the cases, the blazed grating is written to the modulator and the corresponding intensity of the zero and of the first diffracted orders is measured as a function of the time by using the diffraction based set-up given in Fig. 5 and by illuminating the PALCoS display with a He-Ne laser $(633 \mathrm{~nm})$. The period of the grating is fixed to 16 pixels, being the sufficient number of pixels to neglect the effect of the quantification of the phase levels.

The measurements obtained for the intensity diffracted to the zero (in blue) and to the first (in red) orders are plotted in Fig. 13. In Fig. 13(a) and Fig. 13(b), we have plotted the results obtained when using the sequence \#1 and \#2 respectively. Next, Fig. 13(c) and Fig. 13(d) show the results obtained when using the sequence \#3, the former when applying the linear mismatching and the latter when applying the saturation mismatching encoding.

The largest intensity fluctuations are measured when addressing the sequence \#1 (Fig. 13(a)) and the best diffraction efficiency is obtained for the sequence \#3 with the saturated encoding (Fig. 13(d)). In this way, the sequence \#3, even providing a phase modulation lower than 360 degrees (see Fig.11), is the most stable and efficient addressing sequence.

The results here provided evidences that to maximize the efficiency of digital holograms generated with LCoS displays, it is important to find a trade-off between the phase modulation depth and the amplitude of the time-fluctuations phenomena in LCoS displays. In this framework, a mathematical model suitable to evaluate the LCoS display response in presence of time-fluctuations on the phase becomes helpful (Lizana et al., 2010). 

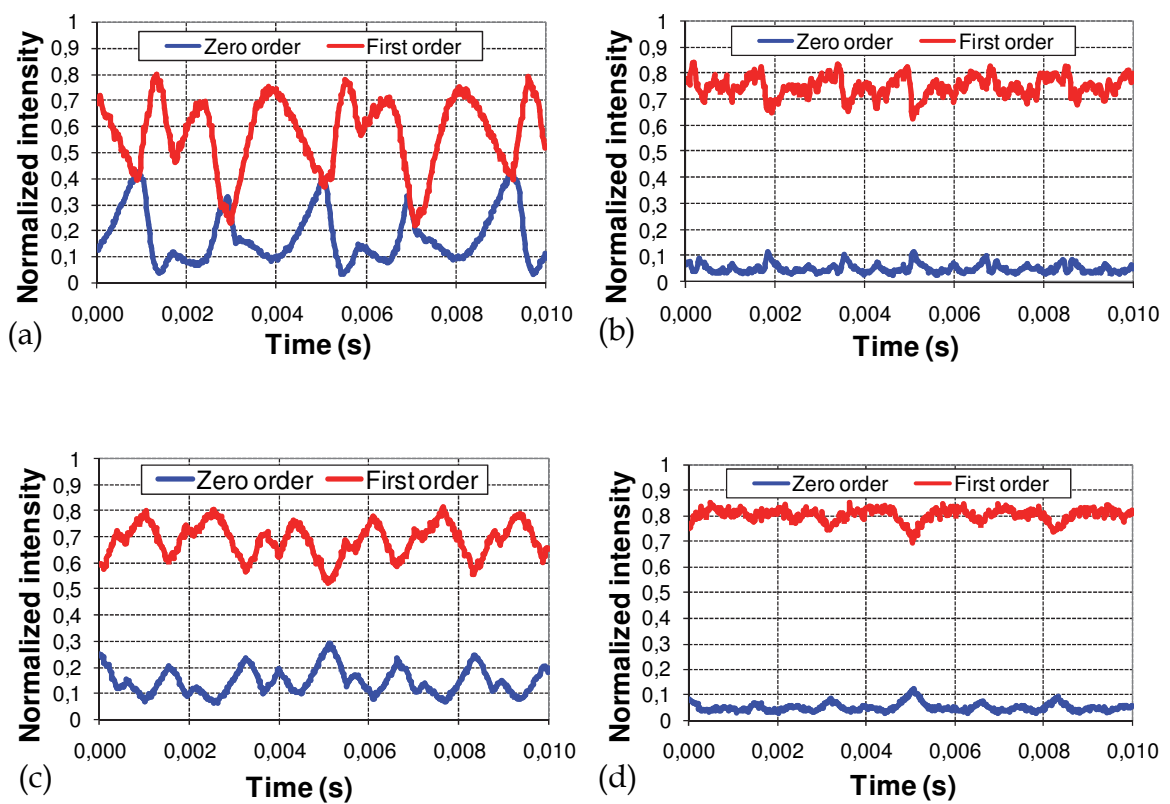

Fig. 13. Normalized intensity for the zero and first orders obtained when addressing a blazed grating and the addressing sequence: (a) \#1, (b) \#2, (c) \#3 with linear mismatch, and (d) \#3 with saturated mismatch.

\section{Conclusions}

This Chapter provides a study of LCoS displays for their application in the generation of digital holograms. In particular, the analysis presented in this Chapter can be used as a guideline to maximize the efficiency of LCoS display in digital holographic applications.

In particular, this Chapter provides a characterization and optimization method, based on a combination of the Mueller formalism and the Jones formalism, suitable to maximize the efficiency of the addressed holograms. Besides, experimental evidence is provided for the time-fluctuations of the phase phenomenon, which degrades the performance of LCoS displays. In this way, a discussion of the damaging effect of such phenomenon, when generating digital holograms, is included. Finally, we experimentally prove that, to maximize the efficiency of digital holograms generated with LCoS displays in presence of phase fluctuations, is important to find a trade-off between phase modulation depth and amplitude of the time-fluctuations. To this aim, different mapping schemes for phase-only distribution implementation are reviewed.

\section{Acknowledgment}

We acknowledge financial support from the Spanish Ministerio de Educación y Ciencia (grants FIS209-13955-C02-01 and FIS209-13955-C02-02). C. Iemmi gratefully acknowledges the support of the Universidad de Buenos Aires and CONICET (Argentina). 


\section{References}

Ashkin, A. (2006). Optical Trapping and Manipulation of Neutral Particles Using Lasers: A Reprint Volume With Commentaries, World Scientific Publishing Company, ISBN 9810240589, Hackensack, USA.

Azzam, R.M.A. \& Bashara, N.D.N. (1972). Ellipsometric Measurement of the Polarization Transfer Function of an Optical System. Journal of the Optical Society of America, Vol. 62, No. 3, pp. 336-340, ISSN 0030-3941.

Berreman, D.W. (1972). Optics in Stratified and Anisotropic Media: 4x4-Matrix Formulation, Journal of the Optical Society of the America, Vol. 62, No. 4, pp. 502-510, ISSN 00303941.

Coufal, H.J.; Psaltis, D. \& Sincerbox, B.T. (2000). Holographic Data Storage, Springer-Verlag, ISBN 0470749628, Berlin, Germany.

Chipman, R. A. (1995); Polarimetry, in Handbook of Optics, McGraw-Hill, New York, USA.

Davis, J.A.; Moreno, I. \& Tsai, P. (1998), Polarization eigenstates for twisted-nematic liquidcrystal displays, Applied Optics, Vol. 37, No. 5, pp. 937-945, ISSN 0003-6935.

De Martino, A.; Kim, Y.; Garcia-Caurel, E.; Laude, B. \& Drévillon, B. (2003). Optimized Mueller polarimeter with liquid crystals, Optics Letters, Vol. 28, No. 8, pp. 616-618, ISSN 0146-9592.

Dickey, F.M.; Holswade, S.C. \& Shealdy, D.L. (2005). Laser Beam Shaping Applications, CRC Press, ISBN 0824759419, Albuquerque, USA.

Dou, R. \& Giles, M.K. (1995). Closed-loop adaptive optics system with a liquid crystal television as a phase retarder, Optics Letters, Vol. 20, pp. 1583-1585, ISSN 01469592.

Fernández-Pousa, C.R.; Moreno, I.; Bennis, N. \& Gómez-Reino, C. (2000). Generalized formulation and symmetry properties of reciprocal nonabsorbing polarization devices: application to liquid-crystal displays, Journal of the Optical Society of America A, Vol. 17, No. 11, pp. 2074-2080, ISSN 0740-3232.

Foldyna, M.; Garcia-Caurel, E.; Ossikovski, R.; De Martino, A. \& Gil, J.J. (2009). Retrieval of a non-depolarizing component of experimentally determined depolarizing Mueller matrices, Optics Express, Vol. 17, No. 15, pp. 12794-12806, ISSN 1094-4087.

Fujita, T.; Nishihara, H. \& Koyama, J. (1982). Blazed gratings and Fresnel lenses fabricated by electron-beam lithography, Optics Letters, Vol. 7, No. 12, pp. 578-580, ISSN 01469592.

Gagnon, R.J. (1981). Liquid-crystal twist-cell optics, Journal of the Optical Society of America, Vol. 71, No. 3, pp. 348-353, ISSN 0030-3941.

Goldstein, D. (2003). Polarized Light, Mercel Dekker, New York, USA, ISBN 0-8247-4053-X.

Hermerschmidt, A.; Osten, S.; Krüger, S. \& Blümel, T. (2007). Wave front generation using a phase-only modulating liquid-crystal based micro-display with HDTV resolution, Proceedings of SPIE, Vol. 6584, pp. 65840E, ISBN. 978-0-8194-6712-6.

Jones, R. C. (1941). A new calculus for the treatment of optical systems, Journal of the Optical Society of America, Vol. 31, No. 7, pp. 488-493, ISSN 0030-3941. 
Juday, R.D. (2001). Generality of matched filtering and minimum Euclidean distance projection for optical pattern recognition, Journal of the Optical Society of America A, Vol. 18, No. 8, pp. 1882-1896, ISSN 0740-3232.

Lee, Y.; Gourlay, J.; Hossack, W.J.; Underwood, I. \& Walton, A.J. (2004). Multi-phase modulation for nematic liquid crystal on silicon backplane spatial light modulators using pulse-width modulation driving scheme, Optics Communications, Vol. 236, pp. 313-322, ISSN 0030-4018.

Liu, H.K.; Davis ,J.A. \& Lilly, R.A. (1985). Optical-data-processing properties of a liquidcrystal television spatial light modulator, Optics Letters, Vol. 10, pp. 635-637, ISSN 0146-9592.

Lizana, A.; Moreno, I.; Iemmi, C.; Márquez, A.; Campos, J. \& Yzuel, M.J. (2008a). Timeresolved Mueller matrix analysis of a liquid crystal on silicon display, Applied Optics, Vol. 47, No. 23, pp. 4267-4274, ISSN 0003-6935.

Lizana, A.; Moreno, I.; Márquez, A.; Iemmi, C.; Fernández, E.; Campos, J. \& Yzuel, M.J. (2008b). Time fluctuations of the phase modulation in a liquid crystal on silicon display: characterization and effects in diffractive optics, Optics Express, Vol. 16, No. 21, pp. 16711-16722, ISSN 1094-4087.

Lizana, A.; Márquez, A.; Moreno, I.; Iemmi, C.; Campos, J. \& Yzuel, M.J. (2008c). Wavelength dependence of polarimetric and phase-shift characterization of a liquid crystal on silicon display, Journal of the European Optical Society - Rapid Publications, Vol. 3, No. 08011, pp. 1-6, ISSN 1990-2573.

Lizana, A.; Martin, N.; Estapé, M.; Fernández, E.; Moreno, I.; Márquez, A.; Iemmi, C.; Campos, J. \& Yzuel, M.J. (2009). Influence of the incident angle in the performance of Liquid Crystal on Silicon displays, Optics Express, Vol. 17, No. 10, pp. 8491-8505, ISSN 1094-4087.

Lizana, A.; Márquez, A.; Lobato, L.; Rodange, Y.; Moreno, I.; Iemmi, C. \& Campos, J. (2010). The minimum Euclidean distance principle applied to improve the modulation diffraction efficiency in digitally controlled spatial light modulators, Optics Express, Vol. 18, No. 10, pp. 10581-10593, ISSN 1094-4087.

Lu, S.Y. \& Chipman, R.A. (1996), Interpretation of Mueller matrices based on polar decomposition, Journal of the Optical Society of America A, Vol. 13, No. 5, pp. 11061113, ISSN 0740-3232.

Márquez, A.; Iemmi, C.; Moreno, I.; Jeffrey, J.A.; Campos, J. \& Yzuel, M.J. (2001), Quantitative prediction of the modulation behavior of twisted nematic liquid crystal displays based on a simple physical model, Optical Engineering, Vol. 40, No. 11, pp. 2558-2564, ISSN 0091-3286.

Márquez, A.; Iemmi, C.; Campos, J.; Escalera, J.C. \& Yzuel, M.J. (2005). Programmable apodizer to compensate chromatic aberration effects using a liquid crystal light modulator, Optics Express, Vol. 13, No. 3, pp. 716-730, ISSN 1094-4087.

Márquez, A.; Moreno, I.; Iemmi, C.; Lizana, A.; Campos, J. \& Yzuel, M.J. (2008). MuellerStokes characterization and optimization of a liquid crystal on silicon display showing depolarization, Optics Express, Vol. 16, No. 3, pp. 1669-1685, ISSN 10944087 . 
Moore, J.R.; Collings, N.; Crossland, W.A.; Davey, A.B.; Evans, M.; Jeziorska, A.M.; Komarcevic, M.; Parker, J.R.; Wilkinson, T.D. \& Xu, H. (2008). The silicon backplane design for an LCOS polarization-insensitive phase hologram SLM, IEEE Photonic Technology, Vol. 20, No. (1/4), pp. 60 - 62.

Moreno, I.; Campos, J.; Gorecki, C. \& Yzuel, M.J. (1995). Effects of amplitude and phase mismatching errors in the generation of a kinoform for pattern recognition, Japanese Journal of Applied Physics, Vol. 34, No. 12 A, pp. 6423-6432, ISSN 0021-4922.

Moreno, I.; Velásquez, P.; Fernández-Pousa, C.R.; Sánchez-López, M.M. \& Mateos, F. (2003). Jones matrix method for predicting and optimizing the optical modulation properties of a liquid-crystal display, Journal of the Applied Physics, Vol. 94, No. 6, pp. 3697-3702, ISSN 0021-8979.

Moreno, I.; Iemmi, C.; Márquez, A.; Campos, J. \& Yzuel, M.J. (2004). Modulation light efficiency of diffractive lenses displayed in a restricted phase-mostly modulation display, Applied Optics, Vol. 43, No. 34, pp. 6278-6284, ISSN 00036935.

Moreno, I.; Lizana, A.; Campos, J.; Márquez, A.; Iemmi, C. \& Yzuel, M.J. (2008). Combined Mueller and Jones matrix method for the evaluation of the complex modulation in a liquid-crystal-on-silicon display, Optics Letters, Vol. 33, No. 6, pp. 627-629, ISSN 0146-9592.

Ossikovski, R.; Anastasiadou, M.; Hatit, S.B.; Garcia-Caurel, E. \& De Martino, A. (2008). Depolarizing Mueller matrices: how to decompose them?, Physica Status Solidi A, Vol. 205, No. 4, pp. 720-727, ISSN 0031-8965.

Parke, N.G. (1949), Optical Algebra, Journal of Mathematics and Physics, Vol. 28, No. 2, pp. 131-139, IDS XT812.

Peinado, A.; Lizana, A.; Vidal, J.; Iemmi, C. \& Campos, J. (2010). Optimization and performance criteria of a Stokes polarimeter based on two variable retarders, Optics Express, Vol. 18, No. 10, pp. 9815-9830, ISSN 1094-4087.

Rodrigo, J.A.; Alieva, T.; Cámara, A.; Marínez-Matos, O.; Cheben, P. \& Calvo, M.L. (2011). Characterization of holographically generated beams via phase retrieval based on Wigner distribution projections, Optics Express, Vol. 19, No 7, pp. 6064-6077.

Taylor, P. (1974). Theory and Applications of Numerical Analysis, Academic Press, ISBN 0-12553560-0, London, United Kingdom.

Twietmeyer, K.M.; Chipman, R.A.; Elsner, A.E.; Zhao, Y. \& VanNasdale, D. (2008). Mueller matrix retinal imager with optimized polarization conditions, Optics Express, Vol. 16, No. 26, pp. 21339-21354, ISSN 1094-4087.

Verma, R.S.; Swami, M.K.; Manhas, S.S. \& Gupta, P.K. (2010). Mueller matrix-based optimization of reflective type twisted nematic liquid crystal SLM at oblique incidences, Optics Communications, Vol. 283, No. 12, pp. 2580-2587, ISSN 0030-4018.

Wolfe, J.E. \& Chipman, R.A. (2006). Polarimetric characterization of liquid-crystal-onsilicon panels, Applied Optics, Vol. 45, No. 8, pp. 1688-1703, ISSN 00036935.

Wu, S.T. \& Yang, D.K. (2005). Reflective Liquid Crystal Displays, John Wiley \& Sons Inc., Chichester, United Kingdom. 
Zhang, Z.; Lu, G. \& Yu, F.T.S. (1994). Simple method for measuring phase modulation in liquid crystal television, Optical Engineering, Vol. 33, No. 9, pp. 3018-3022. 
ADVANCED HOLOGRAPHY METROLOGY AND IMAGING

Edace by trabele Napdanova

\author{
Advanced Holography - Metrology and Imaging \\ Edited by Dr Izabela Naydenova
}

ISBN 978-953-307-729-1

Hard cover, 374 pages

Publisher InTech

Published online 09, November, 2011

Published in print edition November, 2011

Advanced Holography - Metrology and Imaging covers digital holographic microscopy and interferometry, including interferometry in the infra red. Other topics include synthetic imaging, the use of reflective spatial light modulators for writing dynamic holograms and image display using holographic screens. Holography is discussed as a vehicle for artistic expression and the use of software for the acquisition of skills in optics and holography is also presented. Each chapter provides a comprehensive introduction to a specific topic, with a survey of developments to date.

\section{How to reference}

In order to correctly reference this scholarly work, feel free to copy and paste the following:

Angel Lizana, Laura Lobato, Andrés Márquez, Claudio lemmi, Ignacio Moreno, Juan Campos and María J. Yzuel (2011). Study of Liquid Crystal on Silicon Displays for Their Application in Digital Holography, Advanced Holography - Metrology and Imaging, Dr Izabela Naydenova (Ed.), ISBN: 978-953-307-729-1, InTech, Available from: http://www.intechopen.com/books/advanced-holography-metrology-and-imaging/study-ofliquid-crystal-on-silicon-displays-for-their-application-in-digital-holography

\section{INTECH}

open science | open minds

\section{InTech Europe}

University Campus STeP Ri Slavka Krautzeka 83/A 51000 Rijeka, Croatia Phone: +385 (51) 770447

Fax: +385 (51) 686166 www.intechopen.com

\section{InTech China}

Unit 405, Office Block, Hotel Equatorial Shanghai No.65, Yan An Road (West), Shanghai, 200040, China 中国上海市延安西路65号上海国际贵都大饭店办公楼 405 单元 Phone: +86-21-62489820

Fax: $+86-21-62489821$ 
(C) 2011 The Author(s). Licensee IntechOpen. This is an open access article distributed under the terms of the Creative Commons Attribution 3.0 License, which permits unrestricted use, distribution, and reproduction in any medium, provided the original work is properly cited. 